\title{
El debate sobre caridad y derecho en la asistencia social mexicana. Caminos y opciones para una política social
}

\author{
Reseña de: GuadarRama, Gloria (2001), \\ ENTRE LA CARIDAD Y EL DERECHO. \\ UN ESTUDIO SOBRE EL AGOTAMIENTO DEL MODELO NACIONAL DE \\ asistencia social, El Colegio Mexiquense, a.c./Consejo Estatal \\ de Población, Zinacantepec, MéXico, 295 Pp.
}

"La sociedad caritativa se basa en el principio de benevolencia, y la sociedad del bienestar en el principio del derecho. Una sociedad que asiste a los necesitados por el derecho que éstos tienen a la asistencia es menos humillante, en principio, que una sociedad basada en la benevolencia."

Avishai Margalit

Es frecuente que cuando a alguna persona se le inquiere sobre el significado de la asistencia social, siempre la asocia con una actitud, en el mejor de los casos, relacionada con la caridad; esta visión sobre lo asistencial ha estado tan permeada en nuestra sociedad que al parecer no hemos alcanzado a dimensionar que, si la sociedad y los gobiernos no resolvemos pronto el hecho de que los grupos sociales más desfavorecidos no tengan los mínimos de bienestar, no solamente estaremos incurriendo en una grave omisión ética, sino tal vez en un conflicto social de grandes dimensiones.

Por ello he querido comenzar este escrito con el epígrafe inicial del libro Entre la caridad y el derecho, de la investigadora de El Colegio Mexiquense, Gloria Guadarrama. Se trata de una investigación que no puede dejar de ser leída, discutida y analizada por todos aquellos que desde diferentes ámbitos del quehacer profesional, académico o del servicio público, se abocan a estudiar o se interesan por la asistencia social.

El libro de reciente publicación nos introduce en un conjunto de reflexiones en torno a las formas en que hemos percibido y atendido la pobreza en nuestro país, y al desarrollo de las políticas de asistencia pública en el ámbito nacional. En estas 
páginas comentaré algunos puntos relevantes de esa discusión, sobre la base del reconocimiento de la necesidad de alcanzar mayor claridad, desde la óptica del Estado, no solamente sobre las formas de ejecutar y desarrollar la asistencia, sino sobre su significado social y su incidencia en otras políticas sociales.

La autora señala en la presentación que la asistencia social ha sido un tema poco estudiado en México; yo agregaría que en el ámbito regional y en el de las entidades federativas se carece casi por completo de estudios sobre el tema. Esta ausencia se presenta tanto en el medio académico, en particular en la investigación en ciencias sociales, como en el ámbito gubernamental, preocupación fundamental de la autora. Como ella misma señala:

[...] la asistencia social ha sido erróneamente percibida como un ámbito de la acción gubernamental poco importante, quedando al margen de la investigación sistemática y sin tomar en cuenta el papel que ha desempeñado en las formas asumidas por otras políticas sociales y su función legitimadora del régimen político. (p. 13)

Aseveración por demás pertinente en estos tiempos, en los cuales parecería que la reflexión hecha a lo largo de este libro, en el sentido del evidente agotamiento del modelo nacional de asistencia social, no es tomada en consideración, ya que la sociedad en su conjunto y los gobiernos parecen estar empantanados en concepciones, visiones y formas de atender a los pobres y a los sectores vulnerables, que datan de la tradición decimonónica mexicana o de la caridad cristiana.

La lectura de esta obra me ha encaminado a formular algunas preguntas: ¿Serán los goles por la educación una manera eficiente de dar asistencia social y combatir el rezago educativo en ciertas áreas del país? ¿Será la recaudación de fondos a través de eventos en el alcázar del Castillo de Chapultepec un mecanismo idóneo para combatir la pobreza? ¿O es preferible inicialmente contar con una política pública de asistencia social y debatir cuáles son las responsabilidades de la sociedad y de los gobiernos respecto de esta problemática?

Me parece que Gloria Guadarrama nos alerta:

[...] la asistencia social seguirá siendo un imperativo; incluso si continúa siendo segregada y estigmatizada. Los desafíos que plantea el futuro inmediato, para disminuir y aliviar las dimensiones e intensidad de la pobreza, hacen imprescindible revalorar el desinterés con que los mexicanos hemos contemplado a la asistencia; aceptando que su indefinición, su carácter marginal y su insuficiencia expresan el consentimiento y la tolerancia de la sociedad hacia formas inaceptables de des- 
igualdad y discriminación, resumidos en el desinterés por los pobres.

(p. 269)

La relevancia del tema es de primer orden en las agendas nacional y estatal; porque está en relación directa con las dimensiones de la pobreza y con la orientación que pueda asumir la política social en nuestro país.

La obra nos permite entrar a uno de los caminos que nos falta recorrer como sociedad y gobierno en torno a la asistencia social, ya que ésta todavía tendría posibilidades, según nuestra autora, de desempeñar un papel fundamental en la tarea de disminuir los efectos nocivos que sobre los considerados 'extremadamente pobres' han impuesto los modelos económicos neoliberales, sin caer en un asistencialismo paternalista que suplante la acción ciudadana, sin dejar que este asistencialismo se convierta en 'migajas' que alivien la situación de los más necesitados.

Es así que este libro nos ofrece una revisión de las políticas de asistencia social, las cuales a lo largo de 140 años se han mantenido entre las opciones que el mismo Estado y la sociedad en nuestro país han considerado como válidas para aliviar la pobreza.

La investigación está organizada en tres partes:

- La primera establece los elementos desde los cuales se infiere que es posible trazar rasgos, construir un sistema de referencias y atribuir un sentido a la asistencia social mexicana; se sustenta en la hipótesis inicial de encontrar un origen común en las ideas sobre el Estado, la sociedad civil y la asistencia social; este origen común sería el pensamiento de la modernidad.

- La segunda parte nos ofrece un breve examen histórico del proceso de constitución de la asistencia social como política pública puesta en operación por el gobierno de México, iniciada en la última fase del siglo XIX y consolidada durante el siglo xx, con los gobiernos emanados de la Revolución Mexicana.

- La tercera parte está dedicada a la asistencia social en el estado de México; ésta nos permite observar cómo operaron los servicios asistenciales en esta entidad, en primer lugar a través de una dependencia estatal ligada a un instituto nacional, y posteriormente con la coordinación de un sistema nacional. 
No es propósito de esta reseña llevar a cabo un resumen de los diferentes capítulos de la obra; es preferible que cada lector obtenga sus propias conclusiones y pueda tener un debate particular con los planteamientos de la autora.

De manera personal, en estas líneas me propongo entresacar tópicos de la obra que a mi juicio resultan novedosos y pertinentes para un mejor análisis del tema y para elevar el nivel de su discusión.

De la primera parte, me parece fundamental insistir en la necesidad de debatir sobre el papel del Estado en la asistencia social, sobre cuáles son sus funciones y atribuciones en este ámbito: ¿Puede el Estado, cualquiera que éste sea, deslindarse de sus responsabilidades de brindar los mínimos de bienestar social a su población? ¿Podríamos afirmar que en México el tema de la modernidad está superado? ¿Realmente la sociedad mexicana tiene zanjados sus pendientes y sus problemas, con los ideales y propuestas planteadas por la modernidad?

En los inicios del nuevo milenio, ¿podemos afirmar que, a pesar de que México se considera entre la décima y la decimoquinta economía mundial (según el criterio y la institución que lo adopte), tenemos resuelto el problema de la pobreza y el Estado mexicano tiene claro cómo brindar los mínimos de bienestar y el papel que debe desempeñar la asistencia social?

Al revisar la segunda parte, la cual me parece sumamente novedosa y pionera en el género, me quedo con más interrogantes sobre el tema. Sin embargo, esta revisión me permite también deducir y aseverar que en México prácticamente no han existido políticas públicas en materia asistencial; en todo caso ha habido una serie de acciones y proyectos que no han tenido un eje integrador.

Es claro que no contamos con una definición precisa, con una delimitación de la esfera de competencia del ámbito gubernamental en cuanto a la asistencia social. El recorrido histórico nos da prueba de esta indefinición y lo más grave es que en nuestros días están vigentes concepciones ancladas a la década de los cuarenta del siglo pasado, en donde los alcances del papel del Estado en la tarea asistencial son sumamente limitados.

Ello se muestra en el énfasis que anteriormente se hacía, en el sentido de que la miseria desaparecería porque el desarrollo traería consigo una ampliación de la seguridad social y, en consecuencia, la asistencia no sería necesaria; así, la ayuda estatal se daría sólo en la medida de las posibilidades económicas del go- 
bierno; además, la ayuda sería eventual y la prevención debía acabarse, porque de otro modo se contribuiría a perpetuar el desnivel económico de las clases sociales. Este argumento, con todo y sus diferentes matices, ha sido defendido por actores e instituciones políticas de signo diverso.

En conclusión, el aspecto novedoso de este apartado consiste en que permite al interesado en el tema un acercamiento a cómo en el Estado mexicano se han concebido y operado las políticas de asistencia social; de nueva cuenta, un análisis por demás interesante y poco estudiado.

La tercera parte de la investigación, "La asistencia social en el estado de México", me parece un apartado de estudio y análisis obligatorio para todos aquellos involucrados en las tareas de asistencia social en esta entidad; creo, sin temor a equivocarme, que esta parte será tema de referencia y punto de partida en cualquier análisis que se lleve a cabo en la materia.

Como parte del contexto nacional, el estado de México, como otras entidades federativas no ha reproducido mecánicamente los alcances y limitaciones del modelo federal; coincido con la autora en que en las últimas décadas las políticas públicas de asistencia social han tratado de cumplir dos vías: promover el desarrollo y definirse como políticas de atención a la vulnerabilidad; sin embargo, esto no significa que se haya conformado un sistema de protección asistencial, ni una política de impulso a la autogestión y la promoción del ingreso familiar.

Agregaría, o mejor dicho, dejaría como pregunta si la reciente decisión de separar los servicios de salud del Sistema para el Desarrollo Integral de la Familia en el Estado de México (DIFEM) contribuye a consolidar una política integral de asistencia social.

Finalmente, me gustaría resaltar una de las partes que más me llamaron la atención: “Tendencias al fin de milenio", la cual nos permite captar el tipo de fuerzas sociales que mueven la asistencia y su orientación, y que con diferente matiz están presentes en las políticas gubernamentales del último cuarto de siglo. Me refiero a la acentuación del desplazamiento hacia el individualismo; el desplazamiento de la asistencia desde la esfera estatal pública hacia lo público social y privado; a los procesos de integración-desintegración-integración de las instituciones asistenciales; y al desplazamiento en las orientaciones asistenciales desde una posición universalista, hacia una posición selectiva y focalizada. 
Por último, no me resta sino reiterar que el libro Entre la caridad y el derecho. Un estudio sobre el agotamiento del modelo nacional de asistencia social es punto de partida para todos aquellos que están involucrados en las tareas de asistencia social; sus reflexiones y análisis deberán ser tomados en cuenta, ya sea para dar respuesta o para debatir la elaboración de alguna política pública de asistencia social en México.

Eugenio Martínez

Colegio de Bachilleres del Estado de México Correo-e: eugenio_nevado@hotmail.com 